\title{
A successful management after preterm delivery in a patient with severe sepsis during third-trimester pregnancy
}

\author{
Moni Ra ${ }^{1}$, Myungkyu Kim${ }^{1}$, Mincheol Kim¹, Sangwoo Shim ${ }^{1}$, Seong Yeon Hong ${ }^{2}$ \\ Departments of Internal Medicine and ${ }^{2}$ Obstetrics and Gynecology, Catholic University of Daegu School of Medicine, Daegu, Korea
}

A 33-year-old woman visited the emergency department presenting with fever and dyspnea. She was pregnant with gestational age of 31 weeks and 6 days. She had dysuria for 7 days, and fever and dyspnea for 1 day. The vital signs were as follows: blood pressure 110/70 $\mathrm{mmHg}$, heart rate 118 beats/minute, respiratory rate 28 /minute, body temperature $38.7^{\circ} \mathrm{C}$, and oxygen saturation by pulse oximetry $84 \%$ during inhalation of 5 liters of oxygen by nasal prongs. Crackles were heard over both lung fields. There were no signs of uterine contractions. Chest X-ray and chest computed tomography scan showed multiple consolidations and air bronchograms in both lungs. According to urinalysis, there was pyuria and microscopic hematuria. She was diagnosed with community-acquired pneumonia and urinary tract infection (UTI) that progressed to severe sepsis and acute respiratory failure. We found extended-spectrum beta-lactamase producing Escherichia coli in the blood culture and methicillin-resistant Staphylococcus aureus in the sputum culture. The patient was transferred to the intensive care unit with administration of antibiotics and supplementation of high-flow oxygen. On hospital day 2, hypoxemia was aggravated. She underwent endotracheal intubation and mechanical ventilation. After 3 hours, fetal distress was suspected. Under $100 \%$ fraction of inspired oxygen, her oxygen partial pressure was $87 \mathrm{mmHg}$ in the arterial blood. She developed acute kidney injury and thrombocytopenia. We diagnosed her with multi-organ failure due to severe sepsis. After an emergent cesarean section, pneumonia, UTI, and other organ failures gradually recovered. The patient and baby were discharged soon thereafter.

Keywords: Infectious pregnancy complications; Sepsis; Infection

\section{INTRODUCTION}

Sepsis is a common and important cause of death worldwide [1]. Sepsis during pregnancy accounts for only a very small percentage of all sepsis cases, but it is currently the cause of approximately $10 \%$ of global maternal mortality $[1,2]$. When sepsis does occur during pregnancy, proactive

Received: August 6, 2016, Revised: October 30, 2016 Accepted: Accepted: October 31, 2016

Corresponding Author: Sangwoo Shim, Department of Internal Medicine, Catholic University of Daegu School of Medicine, 33, Duryugongwon-ro 17-gil, Nam-gu, Daegu 42472, Korea

Tel: +82-53-650-4274, Fax: +82-53-621-4106

E-mail: swshim@cu.ac.kr early treatment has a massive effect on survival [3]. However, in practice, early treatment may be lacking because of concerns about complications in the fetus. If the disease progresses to severe sepsis or septic shock, there is a high risk of complications due to hypotension, hypoxemia, cardiac dysfunction, and thrombocytopenia in the mother, which makes it difficult to determine whether and when to deliver. Here, we report our experience of successful management in a patient with severe sepsis during pregnancy by aggressive treatment after preterm delivery.

\section{CASE}

Patient: Female, 33 years old

Copyright (C) 2018 Yeungnam University College of Medicine

This is an Open Access article distributed under the terms of the Creative Commons Attribution Non-Commercial License (http://creativecommons.org/licenses/by-nc/4.0/) which permits unrestricted non-commercial use, distribution, and reproduction in any medium, provided the original work is properly cited. 
Main complaint: Fever and dyspnea for 1 day before visiting the hospital

Obstetric history: 0-0-1-0

Current medical history: The patient was at 31 weeks and 6 days gestation. She had visited a primary medical institution due to dysuria starting 7 days previously and fever and nausea starting 1 day previously. However, the symptoms persisted, and the patient developed dyspnea and hypoxia. She was admitted to the emergency room at our hospital receiving 5 L/min of oxygen through a nasal cannula.

Past and family medical history: No specific medical history.

Initial findings: The patient's vital signs were as follows: blood pressure 110/70 $\mathrm{mmHg}$, heart rate 118 beats/minute, respiratory rate 28 breaths/minute, temperature $38.7^{\circ} \mathrm{C}$, and pulse oxygen saturation $84 \%$ while receiving $5 \mathrm{~L} /$ min oxygen through a nasal cannula. The patient was alert and awake but complained of breathing difficulties and right chest pain. Physical examination showed tenderness over the right costovertebral angle. Auscultation revealed crackles in both lungs and no heart murmurs. A nonstress test showed no evidence of preterm labor.

Laboratory findings: Peripheral blood test results were as follows: white blood cell (WBC) count 1,600/ $\mathrm{mm}^{3}$ (neutrophils 92.4\%), hemoglobin $11.5 \mathrm{~g} / \mathrm{dL}$, platelet count 127,000 / $\mathrm{mm}^{3}$, C-reactive protein $139.7 \mathrm{mg} / \mathrm{L}$, procalcitonin $26.98 \mathrm{ng} /$ $\mathrm{mL}$, and lactic acid $3.0 \mathrm{mmol} / \mathrm{L}$. Blood biochemistry results were as follows: aspartate transaminase (AST) $42 \mathrm{IU} / \mathrm{L}$, alanine transaminase (ALT) $18 \mathrm{IU} / \mathrm{L}$, blood urea nitrogen (BUN) 6.7 $\mathrm{mg} / \mathrm{dL}$, creatinine $0.9 \mathrm{mg} / \mathrm{dL}$, serum $\mathrm{Na} 134 \mathrm{mEq} / \mathrm{L}$, serum $\mathrm{K}$ $4.1 \mathrm{mEq} / \mathrm{L}$, lactate dehydrogenase (LDH) $405 \mathrm{U} / \mathrm{L}$, total protein $6.3 \mathrm{~g} / \mathrm{dL}$, albumin $3.1 \mathrm{~g} / \mathrm{dL}$, and brain natriuretic peptide (BNP) $2,055 \mathrm{pg} / \mathrm{mL}$. In a blood clotting test, prothrombin time was 15.4 seconds (international normalized ratio [INR] 1.21), activated partial thromboplastin time was 41.8 seconds, Ddimer was elevated over $20 \mu \mathrm{g} / \mathrm{mL}$, fibrinogen was $442 \mathrm{mg} / \mathrm{dL}$, and antithrombin-III was $64 \%$. In urinalysis of urine collected using a poly-catheter, protein was $2+$, leukocyte esterase was $3+$, WBC count was $\geq 30 /$ high-power field (HPF), red blood cell count was 20-30/HPF, and bacteria was $1+$. In arterial blood gas analysis, $\mathrm{pH}$ was 7.395, partial pressure of $\mathrm{CO}_{2}$ $\left(\mathrm{PaCO}_{2}\right)$ was $25.4 \mathrm{mmHg}, \mathrm{PaO}_{2}$ was $42.1 \mathrm{mmHg}, \mathrm{PaHCO}_{3}$ was $15.2 \mathrm{mmol} / \mathrm{L}$, and oxygen saturation was $78.8 \%$.

Radiographic findings: In the chest X-ray, consolidations were observed in the right superior, middle, and inferior lobes, and in the left inferior lobe (Fig. 1). In high-resolution computed tomography (HRCT) of the lungs, multiple consolidations were observed in the same areas, accompanied by air bronchograms and interlobular septal thickening (Fig. 2).

Clinical course: Following the diagnosis of community-acquired pneumonia and urinary tract infection (UTI), the patient was administered ceftriaxone and azithromycin. The patient was given high-flow oxygen for the hypoxia. At a fraction of inspired oxygen $\left(\mathrm{FiO}_{2}\right)$ of $80 \%, \mathrm{PaO}_{2}$ was $77.2 \mathrm{mmHg}$, and tachypnea persisted. Therefore, the patient was determined to have severe sepsis and acute respiratory failure and was moved to the intensive care unit (ICU). On the 2nd day of hospitalization, the patient continued to show intermittent dyspnea, had a pulse oxygen saturation of $81-85 \%$, a respiratory rate of 30-35 breaths/minute, and had developed cyanosis; following endotracheal intubation, mechanical ventilation was started. In a fetal ultrasound examination immediately after intubation, the fetal heart rate was 160-170 beats/ minute. After 3 hours of intubation, hypoxemia persisted with $\mathrm{PaO}_{2}$ at $87 \mathrm{mmHg}\left(\mathrm{FiO}_{2}\right.$ 100\%) while maintaining mechanical ventilation. Serum creatinine was elevated at $1.9 \mathrm{mg} / \mathrm{dL}$, urinary output was decreased at $5-10 \mathrm{~mL} / \mathrm{hr}$, and metabolic acidosis worsened because of acute kidney injury, necessitating renal replacement therapy. The patient also showed coagulopathy with a platelet count of $67,000 / \mathrm{mm}^{3}$, prothrombin time of 16.1 seconds (INR 1.28), and activated partial throm-

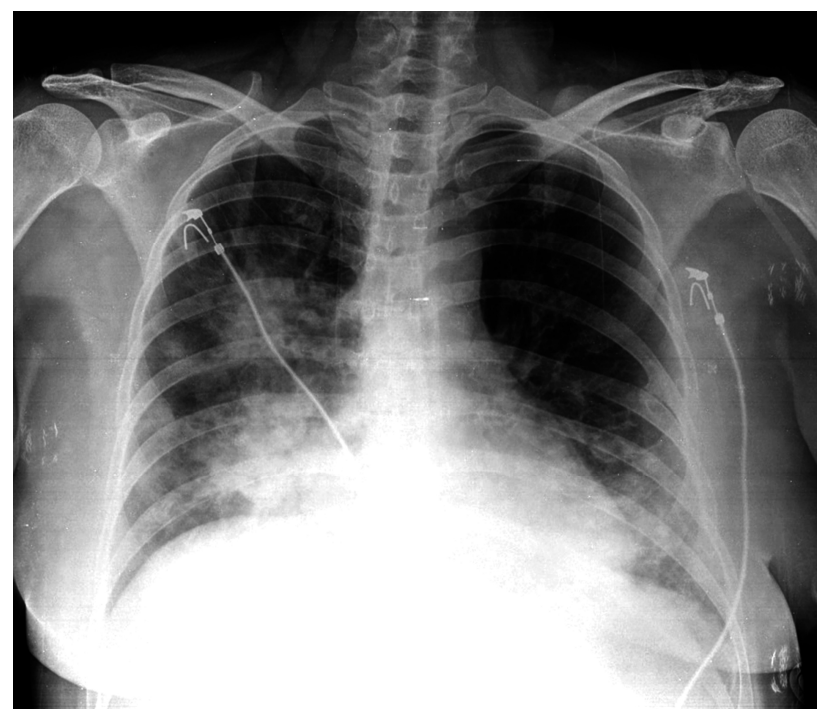

Fig. 1. The initial chest X-ray showed pneumonic consolidations in both lung fields. 
boplastin time of 55.6 seconds. In a nonstress test, the fetal heart rate showed tachycardia at 190-200 beats/minute, and uterine contractions were measured every 2-3 minutes. Fetal heart rate variability was normal, but late decelerations were observed, suggesting fetal distress syndrome. An emergency cesarean surgery was performed. Immediately after delivery, the hourly urinary output began to increase, reaching normal levels 8 hours post-delivery, and systolic blood pressure also increased. Extended-spectrum beta-lactamase (ESBL)-producing Escherichia coli was detected in a blood culture, and methicillin-resistant Staphylococcus aureus (MRSA) was detected in a sputum culture test. On the 5th day of hospitali- zation, the patient's antibiotic regimen was changed to teicoplanin and meropenem, after which her fever began to decline. In spite of pyuria, no bacteria were detected in a urine culture test. On the 9th day of hospitalization, the mother's pneumonia and sepsis improved, and the endotracheal tube was removed. Two days later, the mother was moved to a general ward. On the 16th day post-delivery, the mother was discharged without any complication. The newborn's APGAR score was 4 at 1 minute and 7 at 5 minutes, crying was weak, and activity levels were low. Based on clinical symptoms of respiratory distress syndrome and X-ray findings, an endotracheal tube was placed, and mechanical ventilation was ap-
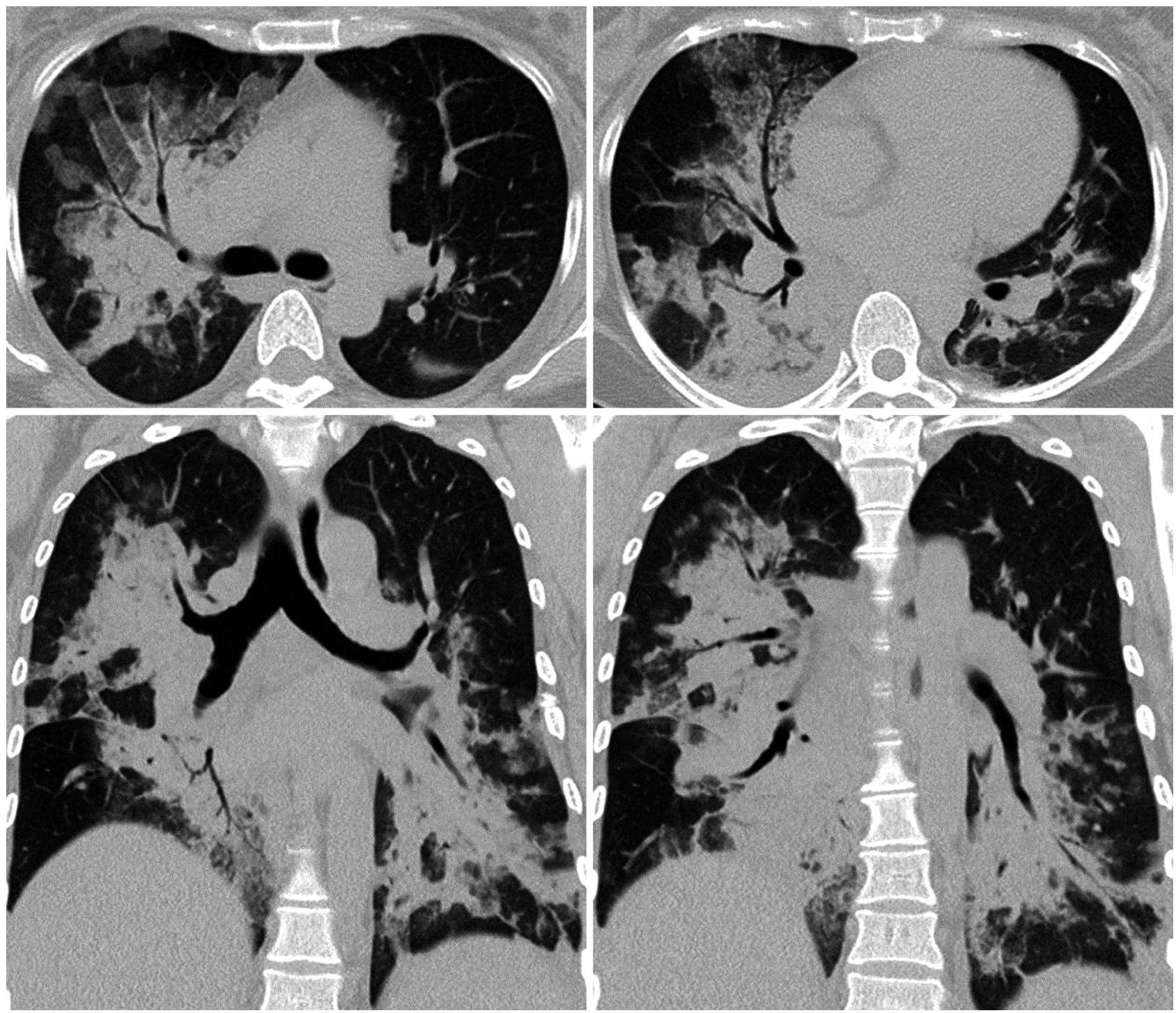

Fig. 2. Chest computed tomography scan showed diffuse patchy consolidations with air bronchograms and mild interlobular septal thickening in both lungs. 
plied while treating the newborn in an incubator. The infant was discharged on the 35th postnatal day with no particular complication.

\section{DISCUSSION}

Sepsis can be diagnosed when infection is confirmed or suspected in a case of systemic inflammatory response syndrome (SIRS). SIRS is defined as the condition satisfying at least two of the following criteria: temperature $<36^{\circ} \mathrm{C}$ or $>38^{\circ} \mathrm{C}$, heart rate $>90$ beats/minute, respiratory rate $>20$ breaths/minute, $\mathrm{PaCO}_{2}<32 \mathrm{mmHg}$, WBC count $<4,000 / \mathrm{mm}^{3}$ or $>12,000 / \mathrm{mm}^{3}$, and immature neutrophils $>10 \%$ [4]. When sepsis is accompanied by failure of at least one organ, severe sepsis can be diagnosed [4]. The present case was diagnosed as severe sepsis on the basis of respiratory failure, acute kidney injury, and coagulopathy. The incidence of sepsis during pregnancy is very low. Septic shock is reported in 0.002$0.01 \%$ of all births, and pregnant women account for only $0.3-0.6 \%$ of all sepsis patients [1,5,6]. Even among pregnant women with bacteremia, only $0-12 \%$ are reported to progress to septic shock [7-9]. On the other hand, among pregnant women admitted to the ICU, the proportion with sepsis is relatively high, with 59\% showing SIRS, 24\% showing severe sepsis, and 3\% showing septic shock [10]. Globally, sepsis accounts for $10 \%$ of maternal mortality [1,2], so it is essential to proactively investigate the cause and provide appropriate treatment. The most common causes of severe sepsis during pregnancy are pyelonephritis and genital infection, and the most common pathogen is E. coli [11]. In the present case, the patient showed symptoms of dysuria, febrile UTI, as well as repeated pyuria. Although bacteria were not observed in the urine culture, it is thought that the UTI was caused by the ESBL-producing E. coli detected in the blood culture. The MRSA detected in the sputum culture could also be considered as causative bacteria for the pneumonia, so UTI and pneumonia are considered to have caused the severe sepsis. Unusually, although this patient was diagnosed with community-acquired pneumonia and UTI, MRSA and ESBL-producing $E$. coli were detected. MRSA is reported to be the causative pathogen in only $10 \%$ of community-acquired pneumonia cases, but this percentage is gradually increasing [12], and such infections are severe, with $75-85 \%$ of patients requiring intensive care and a mortality rate of $20-60 \%$ [13]. Among
UTls caused by $E$. coli, only $1.3 \%$ are reported to be community-acquired infections caused by ESBL-producing $E$. coli. Among cases of UTI caused by ESBL-producing $E$. coli, community-acquired infections are 6-fold more likely than hospital-acquired infections to show no complication and be of mild severity [14]. In this case, the patient may have progressed to severe sepsis due to pneumonia and UTI developing concurrently, and it is impossible to exclude the possibility that ESBL-producing $E$. coli could have been acquired during regular prenatal examinations at a primary medical institution. When pregnant women with sepsis progress to severe sepsis or septic shock, it is important to appropriately judge the timing of delivery. In the present case, there was a high risk of later hemodynamic instability given the high oxygen demand due to respiratory failure, coagulopathy, including thrombocytopenia, and acute kidney injury; however, emergency cesarean surgery also carries an inherent risk, which made it difficult to decide the time of delivery. There are no clear criteria for whether and when to deliver in patients with sepsis. According to the literature, delivery is recommended in pregnant women with severe sepsis or septic shock when accompanied by intrauterine infection, disseminated intravascular coagulation, liver or kidney failure, cardiac arrest, or cardiopulmonary dysfunction (e.g. severe adult respiratory distress syndrome) caused by ascites or the size of the uterus [15]. In the present case, delivery was indicated by disseminated intravascular coagulation, kidney failure, and severe adult respiratory distress syndrome. However, there are numerous cases where delivery is not actually selected despite such indications [3]. In pregnant women being treated for sepsis or severe sepsis, it is important to ensure that delivery happens at an appropriate time, while closely observing not only the state of the underlying disease but also signs of sepsis-related organ failure and fetal distress syndrome. Here, we have reported our experience with a patient who showed severe sepsis, multiple organ failure, and fetal distress syndrome secondary to pneumonia and UTI, where both mother and infant recovered without complications following emergency cesarean surgery and aggressive treatment.

\section{ACKNOWLEDGEMENT}

This study was conducted with intramural research funding from the Catholic University of Daegu in 2014. 


\section{CONFLICT OF INTEREST}

No potential conflict of interest relevant to this article was reported.

\section{ORCID}

Moni Ra, https://orcid.org/0000-0002-0873-176X

Sangwoo Shim, https://orcid.org/0000-0002-9944-2217

\section{REFERENCES}

1. Fernández-Pérez ER, Salman S, Pendem S, Farmer JC. Sepsis during pregnancy. Crit Care Med 2005;33(10 Suppl):S28693.

2. Say L, Chou D, Gemmill A, Tunçalp Ö, Moller AB, Daniels J, et al. Global causes of maternal death: a WHO systematic analysis. Lancet Glob Health 2014;2:e323-33.

3. Snyder CC, Barton JR, Habli M, Sibai BM. Severe sepsis and septic shock in pregnancy: indications for delivery and maternal and perinatal outcomes. J Matern Fetal Neonatal Med 2013;26:503-6.

4. American College of Chest Physicians/Society of Critical Care Medicine Consensus Conference: definitions for sepsis and organ failure and guidelines for the use of innovative therapies in sepsis. Crit Care Med 1992;20:864-74.

5. Martin GS, Mannino DM, Eaton S, Moss M. The epidemiol- ogy of sepsis in the United States from 1979 through 2000. N Engl J Med 2003;348:1546-54.

6. Mabie WC, Barton JR, Sibai B. Septic shock in pregnancy. Obstet Gynecol 1997;90:553-61.

7. Blanco JD, Gibbs RS, Castaneda YS. Bacteremia in obstetrics: clinical course. Obstet Gynecol 1981;58:621-5.

8. Ledger WJ, Norman M, Gee C, Lewis W. Bacteremia on an obstetric-gynecologic service. Am J Obstet Gynecol 1975;121: 205-12.

9. Bryan CS, Reynolds KL, Moore EE. Bacteremia in obstetrics and gynecology. Obstet Gynecol 1984;64:155-8.

10. Afessa B, Green B, Delke I, Koch K. Systemic inflammatory response syndrome, organ failure, and outcome in critically ill obstetric patients treated in an ICU. Chest 2001;120: 1271-7.

11. Acosta CD, Kurinczuk JJ, Lucas DN, Tuffnell DJ, Sellers S, Knight M, et al. Severe maternal sepsis in the UK, 2011-2012: a national case-control study. PLoS Med 2014;11:e1001672.

12. Nakou A, Woodhead M, Torres A. MRSA as a cause of community-acquired pneumonia. Eur Respir J 2009;34:1013-4.

13. Vardakas KZ, Matthaiou DK, Falagas ME. Incidence, characteristics and outcomes of patients with severe community acquired-MRSA pneumonia. Eur Respir J 2009;34:1148-58.

14. Meier S, Weber R, Zbinden R, Ruef C, Hasse B. Extendedspectrum $\beta$-lactamase-producing Gram-negative pathogens in community-acquired urinary tract infections: an increasing challenge for antimicrobial therapy. Infection 2011;39:33340.

15. Barton JR, Sibai BM. Severe sepsis and septic shock in pregnancy. Obstet Gynecol 2012;120:689-706. 